\title{
STUDY ON COUPLING OF SMOOTH PARTICLE METHOD WITH FINITE ELEMENTS BASED ON MESH-PARTICLE MATCHING DEGREE
}

\author{
JUN LIU, PEI WANG \& ANMIN HE \\ Institute of Applied Physics and Computational Mathematics, Beijing, China.
}

\begin{abstract}
In this paper, a new algorithm coupling the finite element and smooth particle methods is proposed. It is an improved algorithm based on the hybrid coupling method. By calculating the mesh-particle matching degree, the new method can better deal with a poor mesh-particle matching relationship. The coupling algorithm can also ensure the transmission of tension and shear stress and the natural disconnection of the contact surface. The comparison of impact simulation shows that the algorithm can deal with mesh-particle separation and re-compaction better, and the coupling interface force is smooth and non-oscillatory. Finally, the new coupling algorithm is applied to the detonation driving problem, and the calculated results are in good agreement with the experimental results.
\end{abstract}

Keywords: coupling method, finite element, smooth particle method, virtual particle.

\section{INTRODUCTION}

In the simulation of multi-material elastic-plastic problems, the finite element (FE) method has the advantages of accurate tracking interface and high computational efficiency, while the smooth particle method has a good description ability for large deformation and fracture. In the study of complex hypervelocity impact dynamics, the phenomena of large deformation, fracture and fragmentation will occur in some regions. Simply the FE method cannot be used to calculate, but we do not want to discard the advantages of the FE method in the less deformed area. In some impact problems, it is suitable to use the finite element-smooth particle coupling method (SPH-FE coupling method). The smooth particle method can be used to analyse the large deformation area, and the FE method to analyse other areas. The interaction can be achieved through a coupling algorithm, thus maintaining the advantages of each method.

At present, the SPH-FE coupling algorithm is mainly composed of several kinds: masterslave method (Attaway et al.[1]); fixed node method (Johnson [2] and Johnson and Holmquist [3]); bridging zone method (Belytschko and Xiao [4] and Wagner and Liu [5]) and hybrid method (Rabczuk et al. [6]). Figure 1 shows the basic ideas of the above coupling algorithms. The SPH-FE coupling method has been successfully applied to the simulation and analysis of complex impact dynamics problems [7-9].

In this paper, based on the hybrid method, the concept of mesh-particle matching degree is introduced, and a new finite element-smooth particle coupling algorithm is developed, which is adapted to change the matching effect in the hybrid method based on mesh-particle matching degree. Compared with the original hybrid method, this algorithm has higher robustness and can effectively reduce the inaccuracy of mesh-particle contact surface calculation caused by the difference of mesh scale-particle radius in initial mesh and particle modelling. Finally, in the calculation of metal cylinder expansion driven by explosive collision, the correctness and effectiveness of the new coupling algorithm are verified by comparing the external diameter of the collision and adjacent parts with the experimental results. 


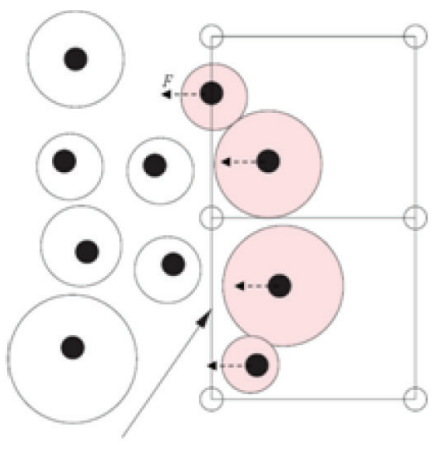

SPH-FE interface

(1) master-slave method

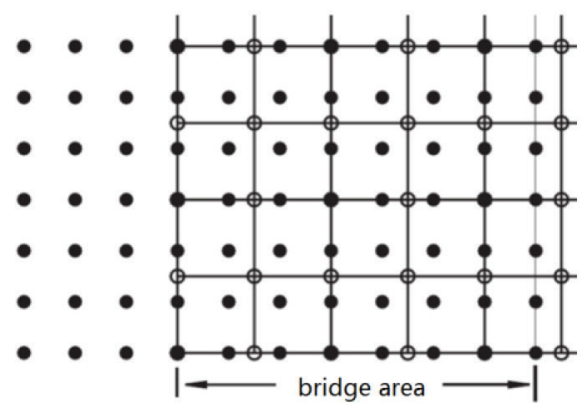

(3) bridging zone method

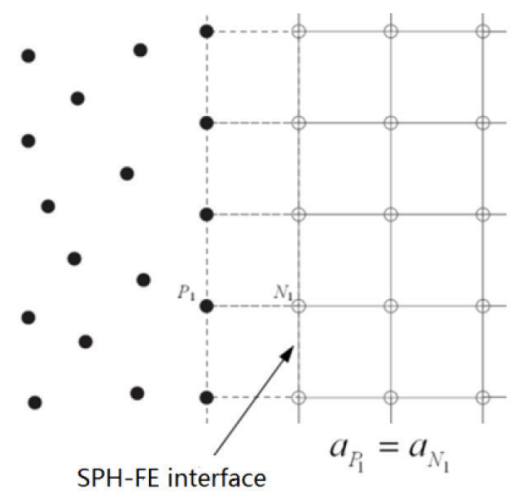

(2) fixed points method

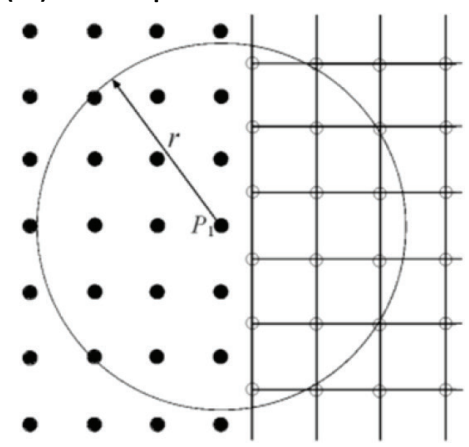

(4) hybrid method

Figure 1: SPH-FE coupling algorithm.

\section{SPH-FE COUPLING ALGORITHM BASED ON MESH-PARTICLE MATCHING DEGREE}

The hybrid method is more flexible and can transfer tension and shear stress at the grid-particle interface, and can handle grid-particle separation. However, Rabczuk et al. [6] and Zhang et al. [8] point out that its shortcoming is that it is not suitable for the grid-particle matching calculation. For example, if only one element node exists in the particle search range, the calculation accuracy will be greatly reduced, and when the mesh width is larger than the particle search diameter, there may be no element node in the particle search range, and the phenomenon of particles penetrating into the mesh may also occur. The coupling algorithm proposed in this paper is an improved hybrid coupling method, which is designed for the case that the hybrid method is difficult to deal with the large difference between mesh-particle sizes.

In order to measure the mesh-particle density, the concept of 'mesh-particle matching degree' is introduced to describe the number of particles on the other side of a Lagrangian boundary. The calculation method of mesh-particle matching is shown in eqns (1) and (2).

$$
\varepsilon_{n}=\frac{L_{\mathrm{n}}}{\overline{S L}_{p}}
$$




$$
\overline{S L}_{p}=\frac{\sum_{R<k L_{\mathrm{n}}} S L_{p}}{m}
$$

where $\varepsilon_{n}$ is the $\mathrm{n}$ edge mesh-particle matching degree, $L_{n}$ is the edge length of $n$ outer surface, and $k$ is the region search coefficient. $\overline{S L}_{p}$ is the average smooth length of $m$ particles in the region with the centre of the edge as the centre of the circle (the centre of the sphere) and the radius of $k L_{\mathrm{n}}$. The radius of the search domain determines the maximum distance between the grid and the particle. Considering that the total time step in the coupled method calculation should ensure that the particle motion does not cross the grid in one time step, the value of $K$ should be in the range 1 to 2 .

Firstly, for each time step, the mesh-particle matching degree on the coupling edges of finite elements is calculated. In Fig. 2, three cases of mesh-particle matching degree calculated according to eqn (1) are given, which are $\varepsilon_{n}=1, \varepsilon_{n}=3, \varepsilon_{n}=6$.

At the second step, according to the mesh-particle matching degree, the mesh on the SPH-FE coupling interface will be transformed into virtual particles. For example, when the matching degree on a coupling edge is $\varepsilon_{n}=3$, the mesh corresponding to the coupling edge will be transformed into $3 \times 3$ virtual particles in the two-dimensional case (see Fig. 3 ). Here, the transformation from mesh to virtual particles can ensure the conservation of mass, momentum and energy.

At the third step, virtual particles are added to SPH real particle calculation. Thus the force of the virtual particle is obtained.

Finally, the force of the virtual particle is summated and distributed on the FE mesh nodes corresponding to the virtual particle according to the distance weighting. Thus a time step calculation is completed.
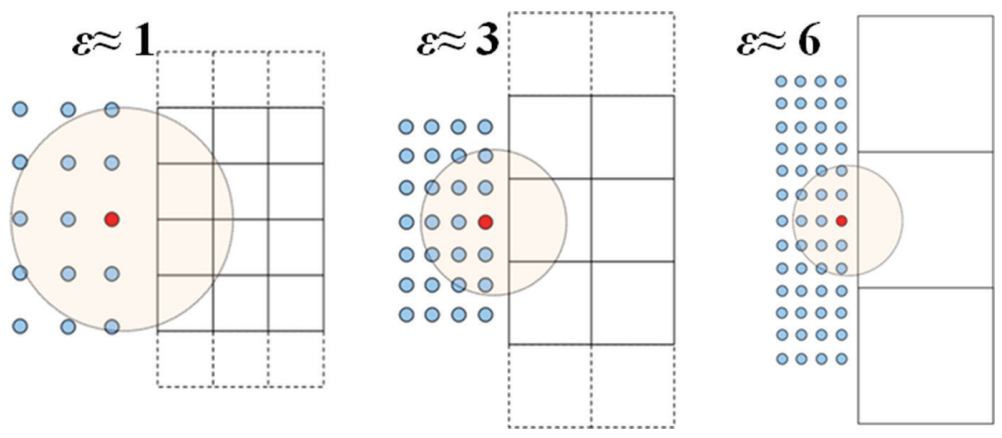

Figure 2: Different mesh-particle matching degrees.
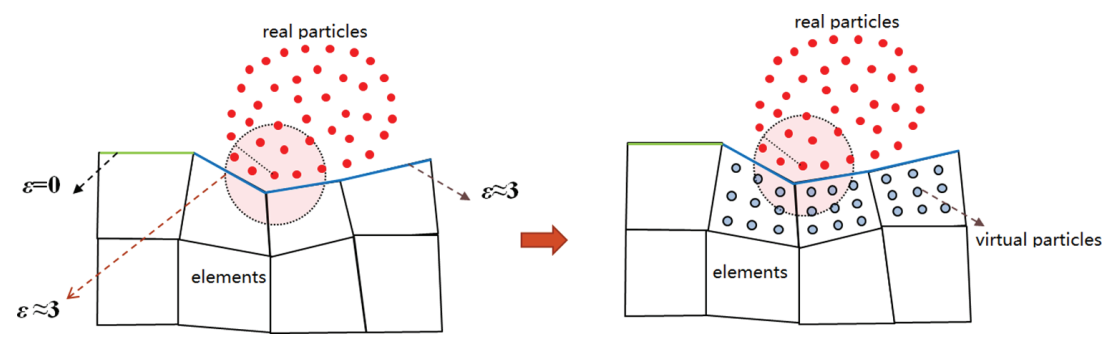

Figure 3: Setting virtual particles based on matching degree on edge. 
Using the above coupling method, the virtual particle acts as the transfer of internal force between FE and SPH method, which is calculated by kernel function in SPH method. Compared with the penalty force method and equilibrium method, the interaction internal force has higher accuracy, and can ensure the transmission of tension and shear stress and the natural disconnection of contact surface.

\section{VALIDATION OF THE NEW COUPLING ALGORITHM}

Firstly, in the case of low-speed collision, the results are compared with those calculated by the simple FE method: an aluminium ball impacts an aluminium target at $500 \mathrm{~m} / \mathrm{s}$ velocity. The mesh and particle generation of the model are shown in Fig. 4a and b. Here, we deliberately divide the particles into denser ones, in order to see whether there will be non-physical phenomena such as particles penetrating the coupling interface.

As shown in Fig. 4c, the improved coupling method in this paper can ensure that particle penetration does not occur. At the same time, the non-physical distortion of local meshes caused by slip line calculation in FE calculation is avoided.

The velocity-time curves of the contact points between sphere and target are shown in Fig. 5.

- The red line is the result of calculation using simple FE method with complete elastic collision slip line algorithm. It is obviously unreasonable that the take-off speed of the single

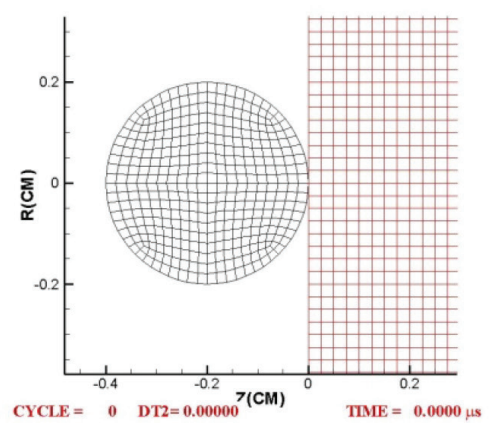

(a) Finite element model

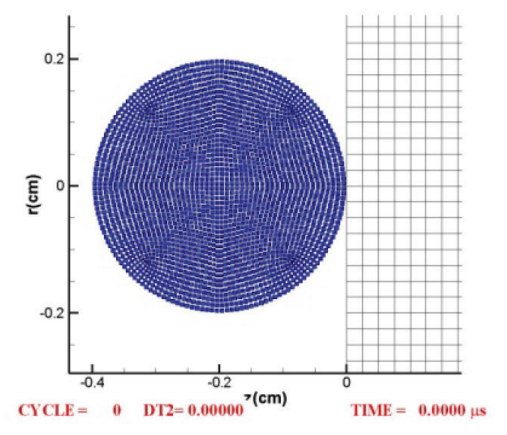

(b) SPH-FE model

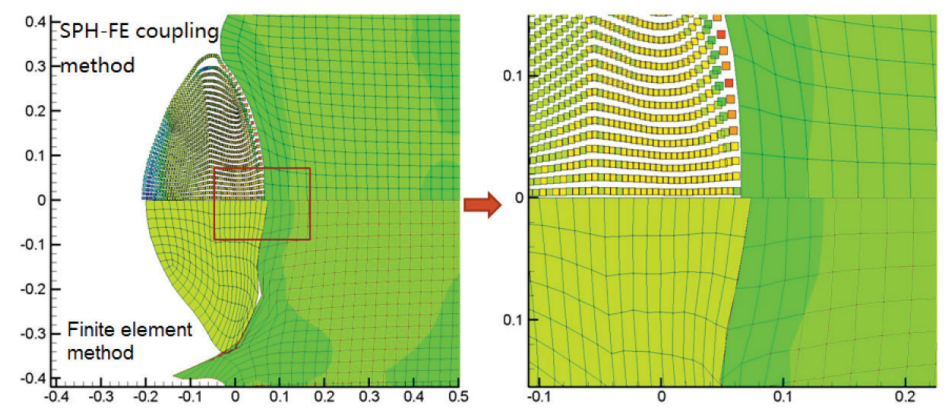

(c) comparisons of calculation results

Figure 4: The computation of high-speed penetration of aluminium balls into aluminium substrates. 


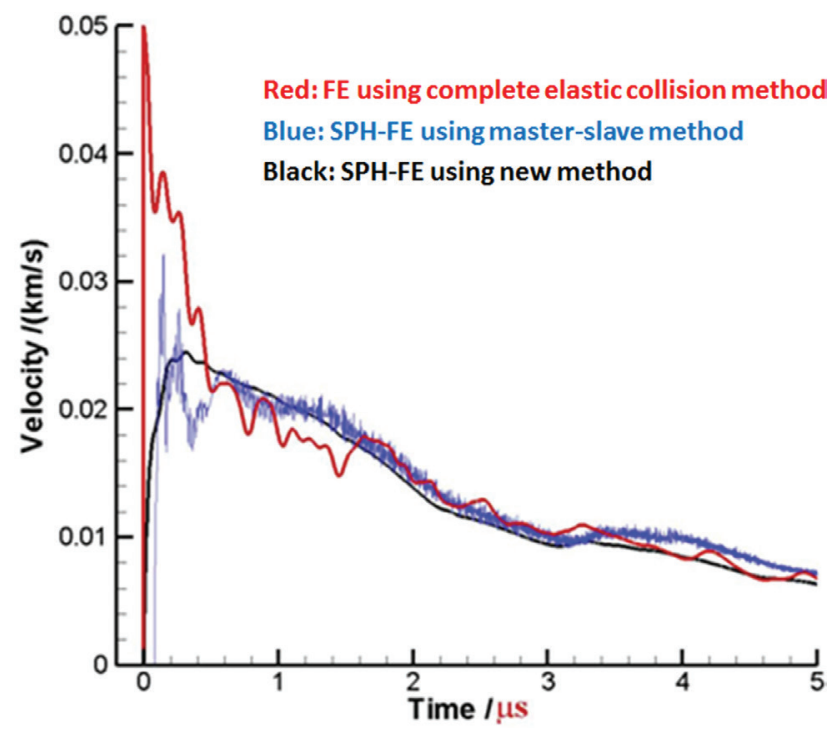

Figure 5: Comparison of velocity-time curves of contact points between sphere and target in different methods.

FE method is $500 \mathrm{~m} / \mathrm{s}$ as that of the aluminium ball due to the use of the slip line treatment of the full elastic collision.

- The blue line is the result of calculation using SPH-FE with master-slave coupling algorithm. Because the master-slave coupling algorithm needs to judge whether the particles invade the unit or not, the method of pushing the particles out after the invasion results in high frequency oscillation of the velocity curve.

- The black line is the result of calculation using the new SPH-FE coupling algorithm. It can capture the take-off speed better, and the speed change is smooth.

In order to verify the correctness of the method, we also compared the results with experimental results. Figure 6a shows a detonation driving setup [10]. HR2 steel shell wrapped explosive JO-9159. The explosive is detonated at both the top and the bottom. The detonation wave collides in the middle of the steel shell, driving the expansion and deformation of the

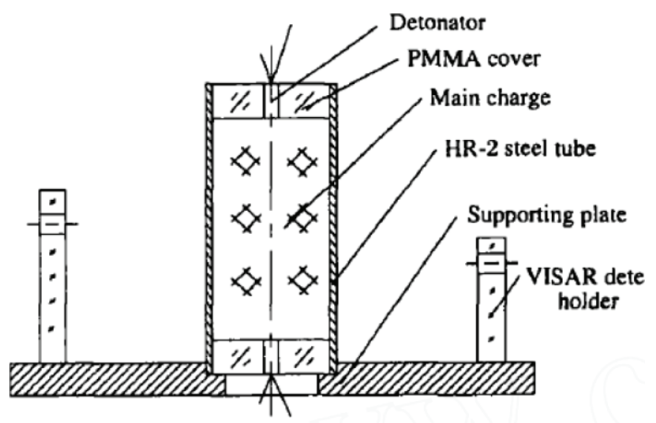

(a) experimental setup 


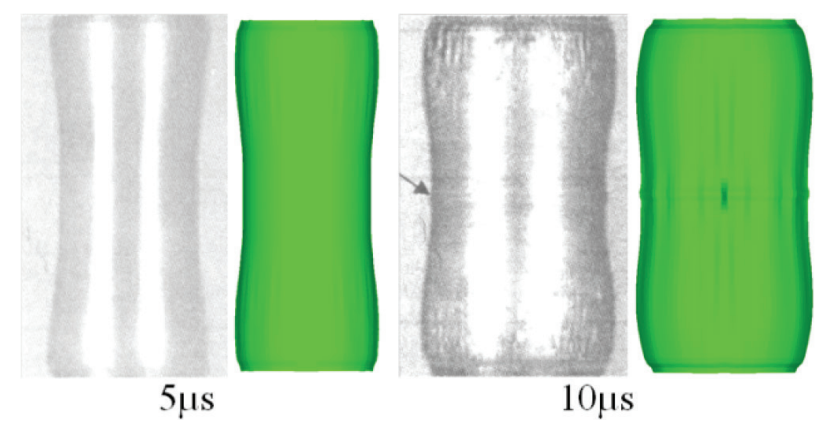

(b) comparisons between X optical image and calculations at $5 \mu \mathrm{s}, 10 \mu \mathrm{s}$

Figure 6: Detonation driving cylinder experiment and SPH-FE calculation.

steel shell. In this example, SPH particles are used to simulate explosive dispersion and FE mesh to simulate the steel shell response; this problem is difficult to be simulated by single FE method or SPH method. The comparison between the X optical image and SPH-FE calculations is shown at $5 \mu \mathrm{s}, 10 \mu \mathrm{s}$ in Fig. $6 \mathrm{~b}$.

Figure 7 shows the SPH-FE calculation results at $17 \mu$ s in the case of simultaneous mesh and particle display. Similarly, no non-physical particle crossing occurred in this example, and the explosive dispersion and shell expansion can be well described. Figure 8 shows the comparison between the experimental and computational results of the position-time image of the detonation collision site, which further verifies the correctness of the improved coupling algorithm in this paper.
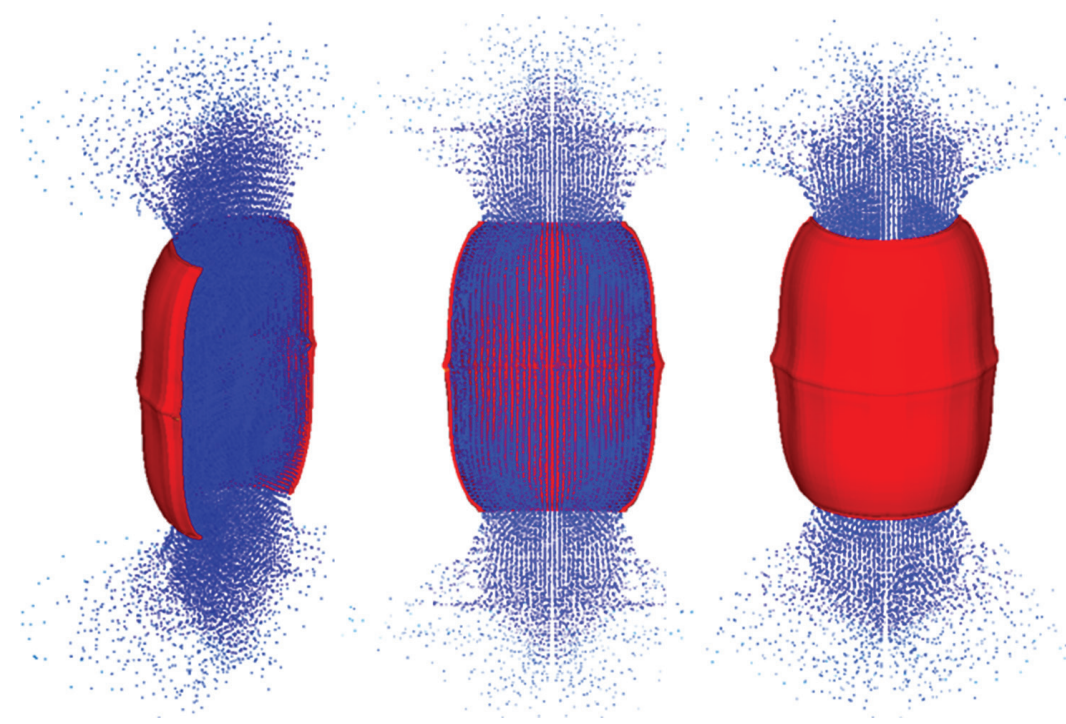

Figure 7: SPH-FE calculation images from three different perspectives at $17 \mu \mathrm{s}$. 

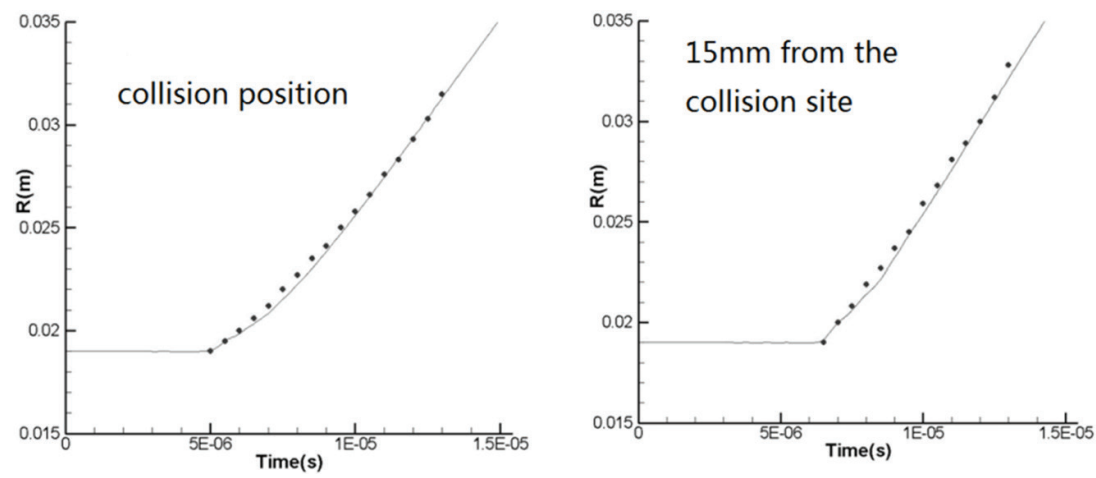

Figure 8: Displacement-Time images of different parts of steel shells (experimental results of circular points).

\section{CONCLUSION}

In order to make the SPH-FE coupling algorithm adapt to the simulation of complex impact dynamics problems and have better robustness and higher coupling accuracy, a finite element smooth particle coupling algorithm based on mesh-particle matching degree is proposed in this paper. The finite element is dynamically mapped to a suitable number of virtual particles by the matching degree of grid particles, and the coupling interaction is realized by the transfer of internal forces by virtual particles.

Tests show that the algorithm can transfer tension and shear stress at the interface, deal with the separation and re-compaction of mesh particles, and has high coupling efficiency, especially for the simulation of complex physical problems at the interface.

\section{ACKNOWLEDGEMENTS}

This study was supported by the YUMIN mathematical foundation (Grant No. TCYM1820-02) and the Science Challenge Project (Grant Nos. TZ2018001 and TZ2016001).

\section{REFERENCES}

[1] Attaway, S.W., Heinstein, M.W. \& Swegle, J.W., Coupling of smoothed particle hydrodynamics with the finite element method. Post-SMIRT Impact IV Seminar, Berlin, Nuclear Engineering and Design, 150(2-3), pp. 199-205, 1994. https://doi. org/10.1016/0029-5493(94)90136-8

[2] Johnson, G.R., Linking of Lagrangian particle methods to standard finite element methods for high velocity impact computations. Nuclear Engineering \& Design, 150(2-3), pp. 265-274, 1994. https://doi.org/10.1016/0029-5493(94)90143-0

[3] Johnson, G.R. \& Holmquist, T.J., Evaluation of cylinder impact test data for constitutive model constants. Journal of Applied Physics, 64(8), pp. 3901-3910, 1988. https:// doi.org/10.1063/1.341344

[4] Belytschko, T. \& Xiao, S.P., A bridging domain method for coupling continua with molecular dynamic. Computer Methods in Applied Mechanics and Engineering, 193, pp. 17-20, 2004. https://doi.org/10.1016/j.cma.2003.12.053

[5] Wagner, G.J. \& Liu, W.K., Coupling of atomistic and continuum simulations using a bridging scale decomposition. Journal of Computational Physics, 190(1), pp. 249-274, 2003. https://doi.org/10.1016/s0021-9991(03)00273-0 
[6] Rabczuk, T., Xiao, S.P. \& Sauer, M., Coupling of mesh-free methods with finite elements: basic concepts and test results. International Journal for Numerical Methods in Biomedical Engineering, 22(10), pp. 1031-1065, 2006. https://doi.org/10.1002/cnm.871

[7] Gui, Y.L., Bui, H.H., Kodikara, J., Zhang, Q.B., Zhao, J. \& Rabczuk, T., Modelling the dynamic failure of brittle rocks using a hybrid continuum-discrete element method with a mixed-mode cohesive fracture model. International Journal of Impact Engineering, 87, pp. 146-155, 2016. https://doi.org/10.1016/j.ijimpeng.2015.04.010

[8] Zhang, Z.C., Qiang, H.F. \& Gao, W.A., A new SPH-FEM coupling method and Its application in impact dynamics. Explosion and Shock, 31(3), pp. 243-249, 2011.

[9] Menouillard, T. \& Belytschko, T., Dynamic fracture with meshfree enriched XFEM. Acta Mechanica, 213(1-2), pp. 53-69, 2010. https://doi.org/10.1007/s00707-009-0275-Z

[10] Zhang, C.Y., Gu, Y., Zhang S.W. \& et al., Expansion and deformation characteristics of metal tubes driven by impact of detonation waves. Explosion and Shock Waves, 25(3), pp. 222-226, 2005. 